\title{
Cross sectional echocardiographic demonstration of the mechanisms of abnormal interventricular septal motion in congenital total absence of the left pericardium
}

Takashi Oki, Tomotsugu Tabata, Hirotsugu Yamada, Kazuyo Manabe, Kazuyo Fukuda, Miho Abe, Arata Iuchi, Nobuo Fukuda, Susumu Ito

\begin{abstract}
Objective-To investigate the influence of the absence of the pericardium on the left ventricular wall, particularly on interventricular septal motion, using $M$ mode and cross sectional short axis echocardiography in patients with congenital total absence of the left pericardium.

Methods-21 patients with congenital total absence of the left pericardium were divided into three groups according to the interventricular septal motion: systolic type $(n=6)$ with paradoxical motion during systole, diastolic type $(n=11)$ with abnormal posterior motion during mid to late diastole, and mixed type $(n=$ 4) with paradoxical motion during systole and abnormal posterior motion during diastole.
\end{abstract}

Results-On cross sectional short axis echocardiograms of the left ventricle, in the diastolic type the degree of angular displacement of the papillary muscles during end diastole to end systole showed excessive anticlockwise rotation about the long axis of the left ventricle without marked anteroposterior displacement. In the systolic type, there was shift of the left ventricle towards the anteromedial portion in systole and towards the posterolateral portion in diastole without significant rotation. There was a significantly positive correlation between the degree of angular displacement and the amplitude of diastolic interventricular septal motion during mid to late diastole in all patients. Conclusions-There was abnormal interventricular septal motion during systole and diastole in patients with total absence of the left pericardium. Abnormal systolic motion was induced by anteroposterior displacement of the left ventricle, and abnormal diastolic motion by left ventricular rotation about the long axis of the heart during the cardiac cycle. Analysis using cross sectional echocardiography was useful for elucidating the mechanisms of abnormal interventricular septal motion.

(Heart 1997;77:247-251)

Keywords: pericardial defect; interventricular septal motion; cross sectional echocardiography
$M$ mode and cross sectional echocardiography have conventionally been used for evaluation of left ventricular wall motion, and these methods have provided important information for detection of regional asynergy of the left ventricular wall in various heart diseases, particularly in myocardial infarction. ${ }^{1}$

Congenital total absence of the left pericardium is a relatively uncommon cardiac malformation. ${ }^{2-11}$ Diagnosis of this abnormality is facilitated by the associated abnormal interventricular septal motion, and the increased use of routine echocardiography. ${ }^{6911}$ The purposes of this study were to investigate alterations of left ventricular position in congenital total absence of the left pericardium and to elucidate the mechanisms of abnormal interventricular septal motion during systole and diastole in this anomaly using $M$ mode and cross sectional echocardiography.

\section{Methods}

STUDY POPULATION

We examined 21 patients (14 men and seven women; mean age 47 (SD 22) years) with congenital total absence of the left pericardium. Diagnosis of the pericardial defect was made by artificial pneumothorax ${ }^{23}$ in 10 patients, and the diagnosis of the condition in the remaining 11 patients was based on the following findings: (1) marked displacement to the left of the heart on a left lateral decubitus chest radiograph ${ }^{2-4}$; (2) $M$ shaped pattern on jugular phlebogram ${ }^{8}$; (3) abnormal interventricular septal motion on $\mathrm{M}$ mode echocardiogram ${ }^{6911}$; and (4) posterolateral displacement of the left ventricle and separation between the aorta and the pulmonary trunk on computed tomography (CT) scans. ${ }^{7}$ All 21 patients underwent routine echocardiography and other clinical examinations, and no other significant complications or cardiovascular abnormalities were observed. Twenty age matched normal volunteers (11 men and nine women; mean age 45 (26) years) served as the control group. The risks, benefits, and alterations in the examinations were explained to all subjects, and informed consent was obtained.

\section{STUDY DESIGN}

Because some reports have shown that interventricular septal motion in this disease may be altered in the lying position, ${ }^{910}$ all echocardiographic studies were recorded with the patient in a left lateral decubitus position. 

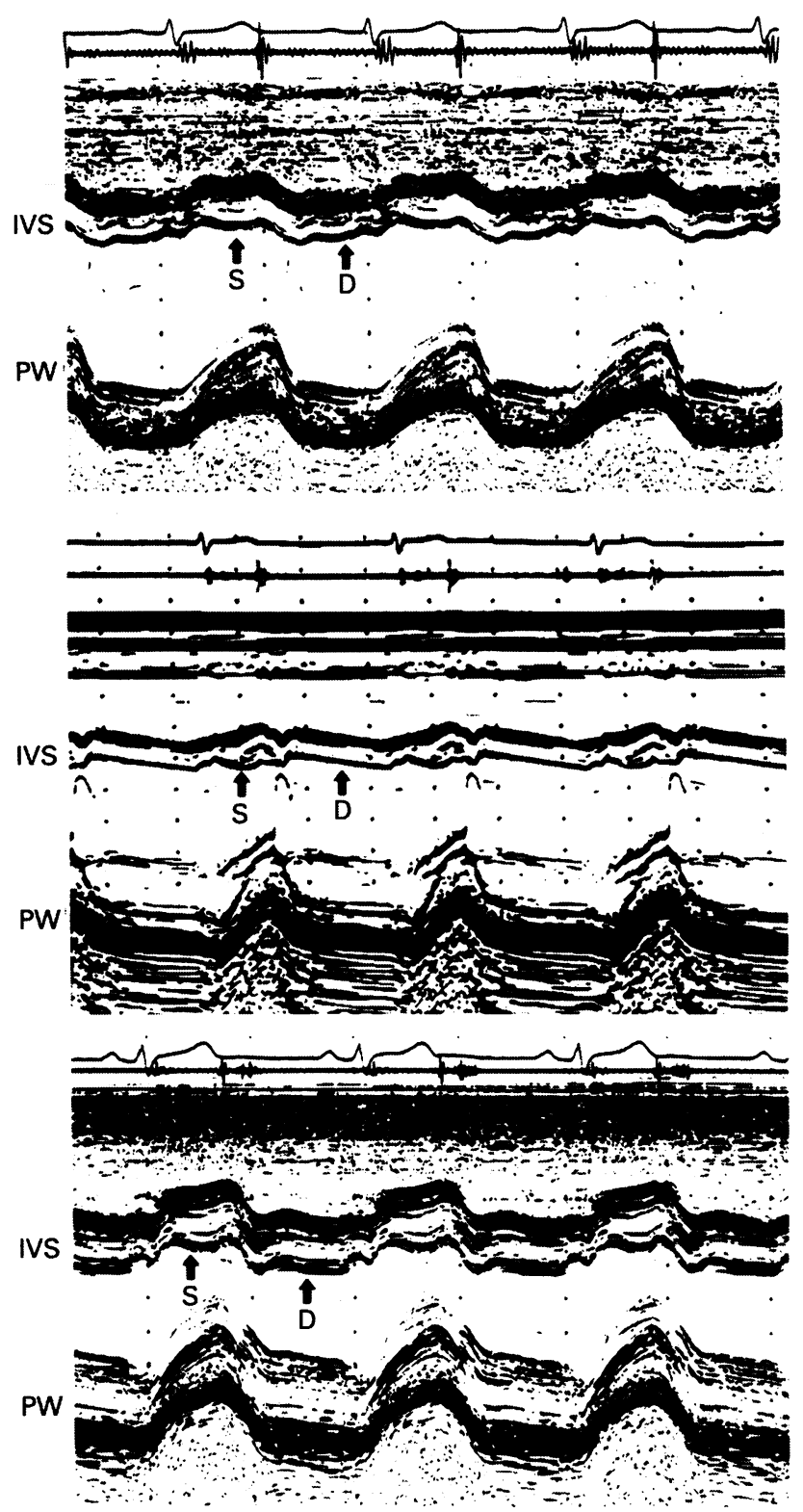

Figure 1 Three types of interventricular septal (IVS) motion in total absence of the left pericardium. Top: Systolic type with paradoxical motion during systole (S) and normal anterior motion during mid to late diastole (D). Middle: Diastolic type with normal posterior motion during systole $(S)$ and abnormal posterior motion during mid to late diastole $(D)$. Bottom: Mixed type with paradoxical motion during systole $(S)$ and abnormal posterior motion during mid to late diastole (D). PW, left ventricular posterior wall.

\section{MODE ECHOCARDIOGRAPHY}

Left ventricular echocardiograms at the level of chordae tendineae were recorded from a parasternal approach. Interventricular septal motion was observed during systole and diastole, and the patterns were divided into the following three types (fig 1): (1) Systolic type: abnormal anterior or paradoxical motion similar to that in the left ventricular posterior wall was observed during systole, but no displacement or normal anterior motion was seen during mid to late diastole; (2) Diastolic type: normal posterior motion was seen during all of systole or early to mid systole, but abnormal posterior motion was seen during mid to late diastole; and (3) Mixed type: both abnormal anterior and posterior motion was observed during systole and mid to late diastole, respectively. A change in interventricular septal motion during mid to late diastole was determined by the amplitude of anterior or posterior motion from the period immediately after the rapid filling phase to end diastole, that is, during the left ventricular slow filling phase (fig 2 , left).

\section{CROSS SECTIONAL ECHOCARDIOGRAPHY}

Cross sectional short axis images of the left ventricle were recorded at the level of the papillary muscles. A line was defined that connected the centres of the anterolateral and posteromedial papillary muscles on the echocardiogram. Angular displacement of that line was measured between end diastole and end systole (fig 2, right). Since angular displacement is considered to reflect left ventricu- $\triangle$ lar rotation about the long axis of the heart, ${ }^{12}$ it $\omega^{\circ}$ was used as an indicator of the degree of clock- $\overrightarrow{0}$ wise or anticlockwise rotation from the perspective of the cardiac apex in this study. All $\vec{\omega}$ cross sectional short axis echocardiograms of the left ventricle in this study were displayed from a perspective overlooking the heart. Therefore the direction of angular displace- $i$ ment on the images was the opposite of that seen from the perspective of the cardiac apex. 0 Cross sectional short axis images of the left ventricle at end diastole and end systole were $\vec{z}$ overlapped using both (1) a reference point on the chest wall and two reference lines of either $\stackrel{5}{工}$ side on the fan shaped image, and (2) displace- $\vec{\theta}$ ment of the interventricular septum and posterior wall; changes in left ventricular shape during the cardiac cycle were then investigated.

Interobserver variability of measurements of $M$ mode and cross sectional echocardiographic $\stackrel{\mathbb{D}}{\circ}$ indices was calculated as the difference in two measurements of the same patient by two dif- 윽 ferent observers divided by the mean value. It was $2.0 \%$ for the $M$ mode echocardiographic index, and $2 \cdot 3 \%$ for the cross sectional echocardiographic index. Intraobserver variability was also calculated as the difference in two measurements of the same patient by one $\frac{5}{3}$ observer divided by the mean value. It was $1.8 \%$ for $M$ mode echocardiographic index, 옳 and $2 \cdot 0 \%$ for cross sectional echocardiographic index.

\section{INSTRUMENTS}

A Toshiba SSH-160A (Toshiba Corporation, N Tokyo, Japan; $2.5 \mathrm{MHz}$ probe) was used for $\mathrm{M}$ N mode and cross sectional echocardiographic N imaging.

\section{STATISTICAL ANALYSIS}

Values are expressed as mean (SD). Mean val- $\mathscr{\Phi}$ ues were compared between groups by analysis 0 of variance (ANOVA) and the Scheffé box test.

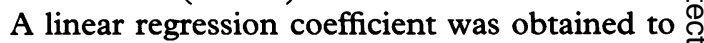
show the degree of correlation between vari- $\mathbb{\mathbb { D }}$ ables. Differences at $P<0.05$ were considered statistically significant.

\section{Results}

$M$ mode and cross sectional echocardiographic variables in 21 patients with pericardial defects and 20 normal controls are summarized in the table. 


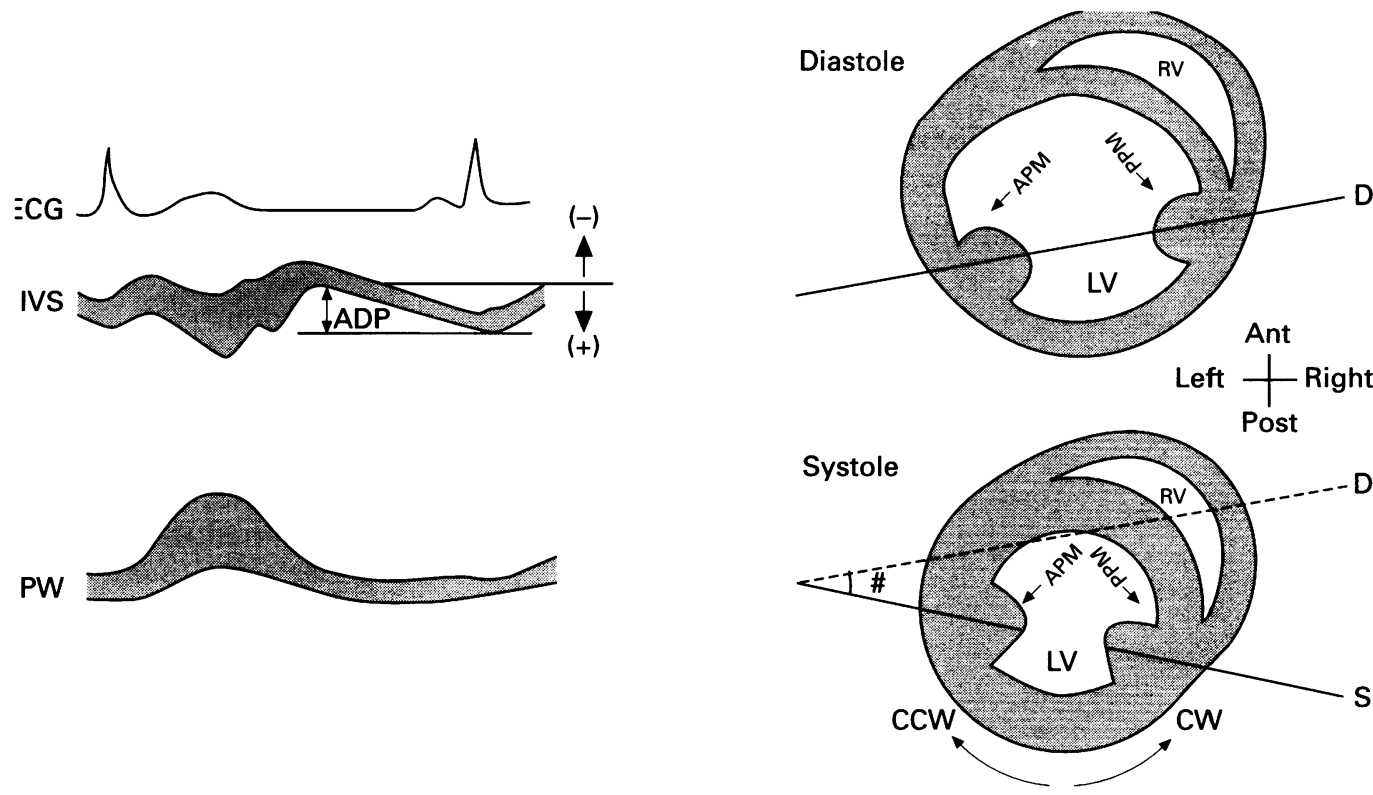

Figure 2 Schematic illustrations of $M$ mode (left) and cross sectional (right) echocardiographic measurements used for this study. Left: Left ventricular echocardiogram at the level of chordae tendineae. Right: Cross sectional short axis echocardiogram of the left ventricle at the level of papillary muscles. A line was drawn connecting the centres of the anterolateral and posteromedial papillary muscles on the echocardiogram. An image from end diastole and one from end systole were superimposed using a reference point from the chest wall, and the angle (\#) between the lines was measured. IVS, interventricular septum; PW, left ventricular posterior wall; ECG, electrocardiogram; $(-)$ and $(+)$, anterior and posterior IVS motions during the left ventricular slow filling phase, respectively; ADP, amplitude of posterior IVS motion during the left ventricular slow filling phase; $R V$, right ventricle; $L V$, left ventricle; $A P M$ and $P P M$, anterolateral and posteromedial papillary muscles, respectively; $C W$ and $C C W$, clockwise and anticlockwise left ventricular rotations about the long axis from the view of the cardiac apex, respectively; $D$ and $S$, lines between the centres of the anterolateral and posteromedial papillary muscle echoes at end diastole and end systole, respectively; \#, degree of the angular displacement of papillary muscles during end diastole to end systole.

Age, $M$ mode, and cross sectional echocardiographic variables in 21 patients with pericardial defect and in 20 normal subjects. Values are mean (SD)

\begin{tabular}{lrlcl}
\hline & $n$ & $\begin{array}{l}\text { Age } \\
\text { (years) }\end{array}$ & $\begin{array}{l}\text { IVS amplitude } \\
(\mathrm{mm})\end{array}$ & $\begin{array}{l}\text { Angular displacement } \\
(\text { degrees) }\end{array}$ \\
\hline Pericardial defect & 21 & $47(22)$ & $2 \cdot 3(1 \cdot 8)^{\star}$ & $10 \cdot 2(7 \cdot 9)^{\star}$ \\
Systolic type & 6 & $44(30)$ & $-0 \cdot 1(0 \cdot 3)$ & $0(2 \cdot 3)$ \\
Diastolic type & 11 & $50(12)$ & $3 \cdot 5(1 \cdot 1)^{\star} \ddagger$ & $15 \cdot 5(4 \cdot 0)^{\star} \ddagger$ \\
Mixed type & 4 & $47(29)$ & $2 \cdot 9(0 \cdot 9)^{\star} \ddagger$ & $11 \cdot 0(6 \cdot 4)^{\star} \ddagger$ \\
Normal control & 20 & $45(26)$ & $-0 \cdot 5(0 \cdot 4)$ & $0 \cdot 8(2 \cdot 2)$ \\
\hline
\end{tabular}

$\star \mathrm{P}<0.0001 v$ normal control; $+\mathrm{P}<0.005, \ddagger \mathrm{P}<0.0001 v$ systolic type

IVS amplitude, amplitude of anterior or posterior interventricular septal motion during the left ventricular slow filling phase; angular displacement, the degree of angular displacement of papillary muscles during end diastole to end systole.

Figure 3 Cross sectional short axis echocardiograms of the left ventricle in two patients with systolic type (top) and diastolic type

(bottom) of pericardial

defects. Top: Left ventricle is displaced excessively towards the anteromedial portion at end systole (middle) and towards the posterolateral portion at end diastole (left), although there is a minimal degree of clockwise left ventricular rotation $\left(3^{\circ}\right)$ during the cardiac cycle. Bottom: Lef ventricle indicates an exaggerated degree of anticlockwise left

ventricular rotation $\left(16^{\circ}\right)$, although there is no

excessive anteroposterior displacement during the cardiac cycle. IVS,

interventricular septum;

$P W$, left ventricular

posterior wall; $A P M$ and

PPM, anterolateral and posteromedial papillary muscles, respectively; $R$ and $L$, right and left sides,
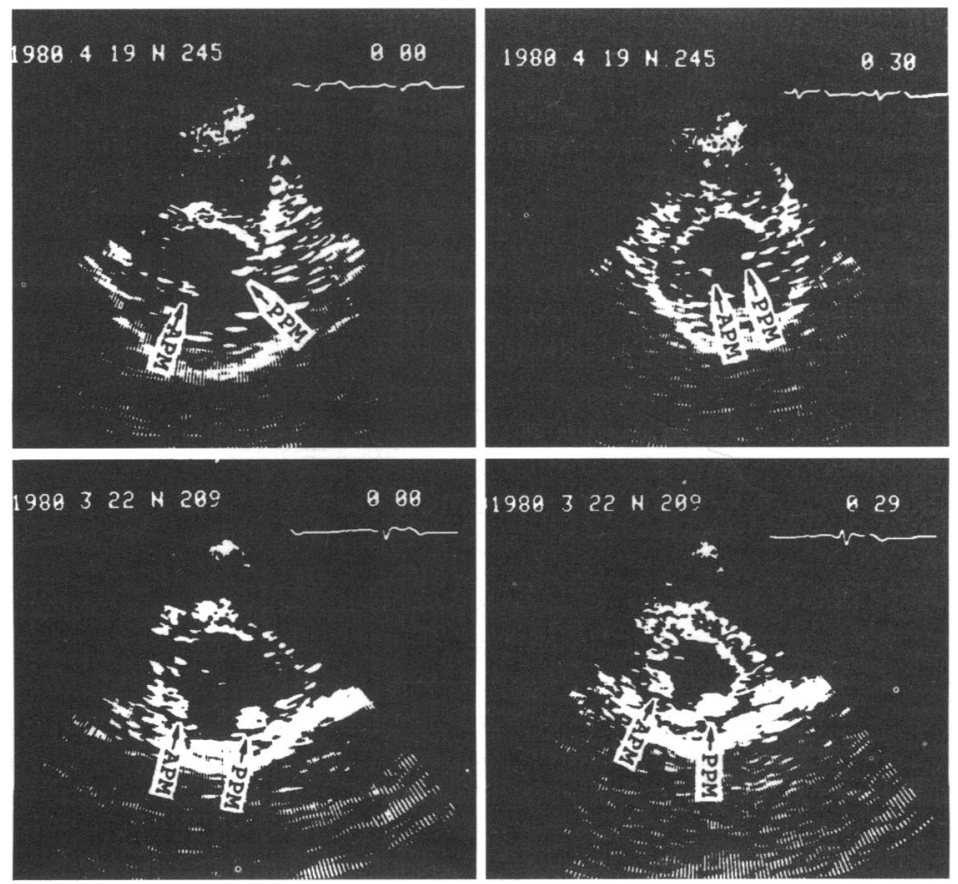

respectively.

INTERVENTRICULAR SEPTAL MOTION

The systolic type of abnormal interventricular septal motion was observed in six of 21 patients, the diastolic type in 11, and the mixed type in four. The amplitude of the interventricular septal motion during mid to late diastole in the diastolic type was significantly greater than in the systolic type $(P<0.0001)$ and in normal subjects $(P<$ 0.0001 ). 


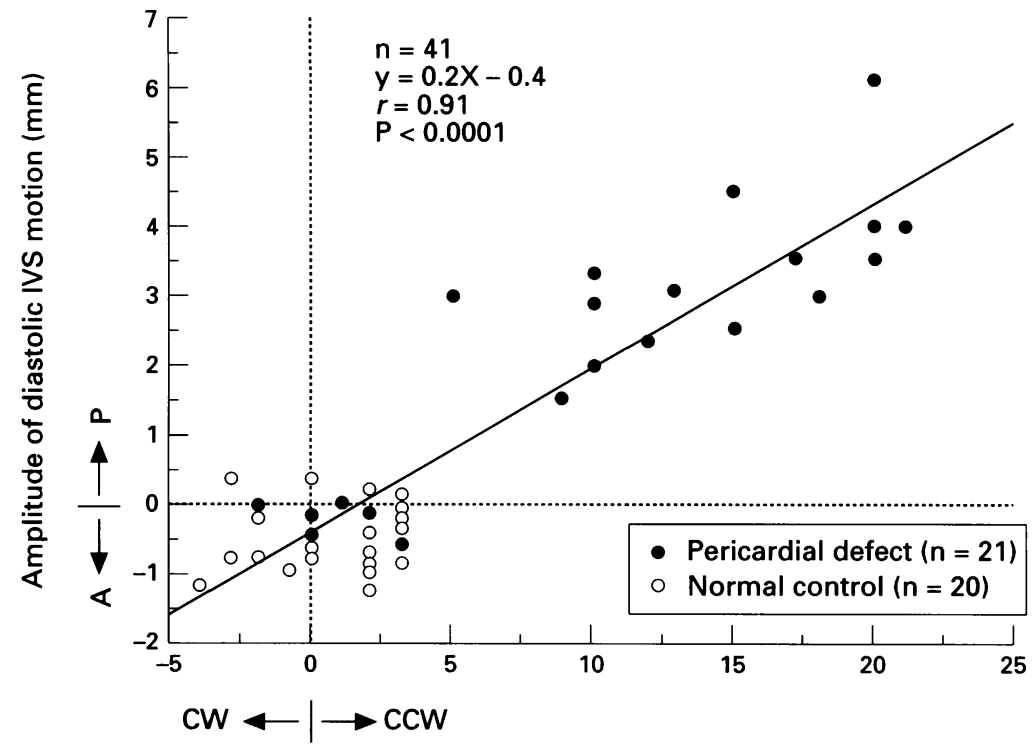

Angular displacement (degree)

Figure 4 Correlation between the amplitude of interventricular septal (IVS) motion during the left ventricular slow filling phase and the degree of angular displacement of papillary muscles during end diastole to end systole in 21 patients with pericardial defect and in 20 normal subjects. $A$ and $P$, anterior and posterior IVS motions during the left ventricular slow filling phase, respectively; $C W$ and $C C W$, clockwise and anticlockwise left ventricular rotations about the long axis from the view of the cardiac apex, respectively.

\section{CROSS SECTIONAL SHORT AXIS}

ECHOCARDIOGRAPHY

The left ventricle maintained a circular shape throughout the cardiac cycle in all 21 patients, similar to that in normal subjects. Short axis images of the left ventricle revealed normal concentric contraction during systole in all 20 normal subjects. However, six patients with systolic type of interventricular septal motion and four patients with the mixed type showed marked anteroposterior motion, in which the left ventricle was excessively displaced towards the anteromedial portion during systole and towards the posterolateral portion during diastole (fig 3, top). The 11 patients with the diastolic type also showed marked anteroposterior motion of the posterior wall during the cardiac cycle, whereas there was no significant displacement of the interventricular septum (fig 3, bottom).

There was exaggerated anticlockwise rotation in angular displacement of the papillary muscles in the diastolic and mixed types compared with those in the systolic type and in normal subjects $(P<0.005$ and $P<0.0001)$. There was a significantly positive correlation ( $r=0.91, \mathrm{P}<0.0001$ ) between the degree of angular displacement of the papillary muscles and the amplitude of interventricular septal motion during mid to late diastole in all patients and normal subjects (fig 4).

\section{Discussion}

Congenital total absence of the left pericardium is a relatively uncommon cardiac malformation. ${ }^{2-11}$ In the past, this anomaly was diagnosed by injecting air into the left pleural space (artificial left pneumothorax). ${ }^{23}$ When the pericardial defect is present on the left side of the heart, this air can enter the pericardial cavity through the defect. In recent years, the diagnosis of this anomaly has been facilitated by the widespread use of echocardiography. ${ }^{6911}$ That is, total absence of the left pericardium is characterised by abnormal systolic interventricular septal motion, although partial absence of the pericardium may not be associ-

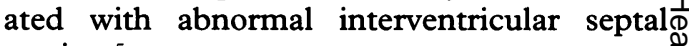
motion. ${ }^{5}$

Other cardiac diseases, particularly atrial septal defect, may show abnormal systolic interventricular septal motion, and must be differentiated from total absence of the left $\frac{\bar{O}}{\bar{D}}$ pericardium. ${ }^{1314}$ Payvandi and Kerber ${ }^{6}$ have $\frac{\widetilde{\sigma}}{\widetilde{\sigma}}$ reported that abnormal systolic interventricu- $\cong$ lar septal motion in total absence of the leftes pericardium is characterised by marked dis- $\overrightarrow{0}$ placement of the whole heart including the leftventricle towards the posterolateral portion. during diastole, and towards the anteromedial? portion during systole. Beppu et $a l^{9}$ have? shown that abnormal interventricular septal. motion changes when patients are in the lying position and that the most characteristic $\_$ motion is induced in the left lateral decubitus응 position.

The absence or presence of cardiac rotation $\bar{Z}$ about its long axis during the cardiac cycle has been investigated using dimensional and variable resistance gauges in dogs, ${ }^{15} 16$ cineangio- $\vec{\bullet}$ graphy with endocardial and epicardial. markers, ${ }^{1718}$ and magnetic resonance (MR)응 imaging. ${ }^{19}{ }^{20}$ In normal subjects, there is only slight anticlockwise rotation $\left(3-7^{\circ}\right)$ about the long axis of the heart. These results suggest that the pericardium immobilises the heart $\stackrel{\mathbb{Q}}{\stackrel{\mathbb{Q}}{2}}$ and thereby controls excessive rotation about $\vec{\overrightarrow{ }}$ its long axis. Consequently, pendular motion 3 of the heart-which mainly includes anteroposterior motion-provides a motivating force? for efficient ejection of blood from the left ventricle.

With regard to left ventricular rotation in atrial septal defect, Ohta $e t a l^{21}$ have observed 3 anticlockwise rotation of the left ventricle during systole when viewed from the cardiac apex on left ventriculography. This was thought to응 be due to posterosuperior compression of the left ventricle by the markedly enlarged righto ventricle. As a result, the interventricular septum become convex towards the left ventricle. during diastole, ${ }^{14}$ resulting in distortion in left ${ }^{N}$ ventricular shape which is never seen in patients with total absence of the left pericardium. Mirro et al ${ }^{12}$ have recorded cross sec-e tional short axis echocardiograms of the left ventricle at the level of the papillary muscles, ? and reported that angular displacement during ${ }_{T}^{0}$ end diastole to end systole in patients with ${ }_{\mathbb{\Phi}}$

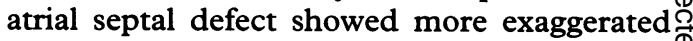
anticlockwise rotation than that in normal $\stackrel{\circ}{\circ}$ subjects. They emphasised that the degree of angular displacement of the papillary muscles? reflected left ventricular rotation about the long axis of the heart.

In this study, patterns of abnormal interventricular septal motion were divided into systolic, diastolic, and mixed types, and the mechanisms of these abnormal motions were investigated. The systolic type, in which 
abnormal anterior interventricular septal motion-that is, paradoxical motion-was observed only during systole, is consistent with the findings reported by Payvandi and Kerber ${ }^{6}$ : (1) cross sectional short axis images of the left ventricle showed displacement of the interventricular septum and posterior wall towards the anteromedial portion during systole and towards the posterolateral portion during diastole, without change in left ventricular shape, and resulted in excessive anteroposterior motion of the left ventricle during the cardiac cycle; and (2) the degree of angular displacement of the papillary muscles showed no significant change during the cardiac cycle. There was no exaggerated rotation about the long axis of the left ventricle in the systolic type, and abnormal motion of the left ventricle due to absence of the left pericardium was derived mainly from anteroposterior motion of the heart. Therefore, left ventricular motion in the systolic type was similar to pendular motion in patients with massive pericardial effusion. $^{22}$ Since pendular motion mainly includes anteroposterior motion of the left ventricle without rotation about its long axis during systole, left ventricular contraction may induce inefficient blood ejection from the left ventricle in the systolic type.

On the other hand, the following characteristic findings were observed in the diastolic type, in which the interventricular septum shows abnormal posterior motion during diastole. (1) Cross sectional short axis images of the left ventricle showed that the left ventricle maintained its circular shape-similar to the systolic type-during the cardiac cycle, but that the interventricular septum had no significant shift despite marked displacement of the posterior wall. (2) The degree of angular displacement of the papillary muscles showed exaggerated anticlockwise rotation during diastole to systole, and it was significantly positively correlated with the amplitude of the diastolic interventricular septal motion on $M$ mode echocardiography.

From these findings, exaggerated anticlockwise rotation about the long axis of the left ventricle during diastole to systole and abnormal posterior interventricular septal motion (clockwise rotation) during mid to late diastole were considered the main motion abnormalities in the diastolic type, despite the absence of excessive anteroposterior motion of the left ventricle. The interventricular septum in the diastolic type showed no significant motion towards the anteromedial portion during systole, but there was marked motion of the posterior wall towards the anteromedial portion. Since exaggerated anticlockwise rotation takes part in left ventricular contraction, blood may be ejected from the left ventricle with relatively high efficiency.

When considering the mixed type, however, the left ventricle is predicted to show various dynamic abnormalities because of the anatomical relation between the extent of the pericardial defect and the position of the heart in the absence of the left pericardium. Therefore, it is impossible to explain the abnormal interventricular septal motion in this anomaly using only the concept proposed by Payvandi and Kerber. ${ }^{6}$

\section{CONCLUSIONS}

Interventricular septal motion during diastole and systole in congenital total absence of the left pericardium was regulated by left ventricular rotation about the long axis of the heart and left ventricular anteroposterior motion, respectively. Analysis by cross sectional echocardiography was useful for evaluating the mechanisms of abnormal interventricular septal motion in this anomaly.

1 Henry WL, DeMaria A, Gramiak R, King DL, Kisslo JA, Popp RL, et al. Report of the American Society of Echocardiography Committee on nomenclature and standards in two-dimensional echocardiography. Circulation 1980;62:212-7.

2 Ellis KE, Leeds NE, Himmelstein A. Congenital deficiencies in the parietal pericardium. A review with two new cases including successful diagnosis by plain roentgenography. Am $\mathcal{F}$ Roentgerzol 1959;82:125-37.

3 Nasser WK, Helmen C, Tavel ME, Feigenbaum H, Fisch C. Congenital absence of the left pericardium: clinical, electrocardiographic, radiographic, hemodynamic, and angiographic findings in six cases. Circulation 1970; 41:469-78.

4 Morgan JR, Rogers AK, Forker AD. Congenital absence of the left pericardium: clinical findings. Ann Intern Med the left pericardi

5 Robin E, Ganguly SN, Fowler MS. Strangulation of the left atrial appendage through a congenital partial pericardial defect. Chest 1975;67:354-5.

6 Payvandi MN, Kerber RE. Echocardiography in congenital and acquired absence of the pericardium: an echocardiographic mimic of right ventricular volume overload. Circulation 1976;53:86-92.

7 Baim RS, MacDonald IL, Wise DJ, Lenkei SC. Computed tomography of absent left pericardium. Radiology 1980; 135:127-8.

8 Matsuhisa $M$, Shimomura $K$, Beppu S, Nakajima K. Jugular phlebogram in congenital absence of the pericardium. Am Heart $\mathcal{f}$ 1986;112:1004-10

9 Beppu S, Matsuhisa M, Izumi S, Masuda Y, Nagata S, Park Y, et al. Pericardial defect: roles of the pericardium on kinetoanatomic changes of the heart influenced by patients' postures. $f$ Cardiogr 1986;16:193-205.

10 Beppu S, Naito H, Matsuhisa M, Miyatake K, Nimura Y.

The effects of lying position on ventricular volume in congenital absence of the pericardium. Am Heart $\mathcal{f} 1990$; congenital absenc.

11 Connolly HM, Click RL, Schattenberg TT, Seward JB, Tajik AJ. Congenital absence of the pericardium: echocardiography as a diagnostic tool. $\mathcal{F} \mathrm{Am}$ Soc Echocardiogr 1995;8:87-92.

2 Mirro MJ, Rogers EW, Weyman AK, Feigenbaum H. Angular displacement of the papillary muscles during the cardiac cycle. Circulation 1979;60:327-33.

13 Diamond MA, Dillon JC, Haine CL, Chang S. Feigenbaum $\mathrm{H}$. Echocardiographic features of atrial septal defect. Circulation 1971;43:129-35.

14 Weyman AK, Wann S, Feigenbaum $H$, Dillon JC. Mechanism of abnormal septal motion in patients with Mechanism of abnormal septal motion in patients with right ventricular volume overload: a cross-sectio

15 Rushmer RF. Physical characteristics of myocardial performance. Am $\mathcal{F}$ Cardiol 1966;18:6-9.

16 Hawthorne EW. Dynamic geometry of the left ventricle. Amorne EW. Dynamic geome

17 Sandler H, Dodge HT. The use of single plane angiocardiograms for the calculation of left ventricular volume in diograms for the calculation of left
man. Am Heart $f$ 1968;75:325-34.

18 McDonald IG. The shape and movements of the human left ventricle during systole. Am $\mathcal{f}$ Cardiol 1970;26: 221-30.

19 Pelc LR, Sayre J, Yun K, Castro LJ, Herfkens RJ, Miller DC, et al. Evaluation of myocardial motion tracking with cine-phase contrast magnetic resonance imaging. Invest Radiol 1994;29:1038-42.

20 O'Dell WG, Moore CC, Hunter WC, Zerhouni EA, McVeigh ER. Three-dimensional myocardial deformations: calculation with displacement field fitting to tagged MR images. Radiology 1995;195:829-35.

21 Ohta $M$, Uehara $T$, Naito $H$, Nishimura $T$, Sugahara $T$, Maeda $\mathrm{H}$, et al. Angiographic evaluation of mitral valve prolapse associated with secundum atrial septal defect. $\mathcal{f}$ Cardiogr 1980;10:53-8.

22 Feigenbaum H, Zaky A, Grabhorn L. Cardiac motion in patients with pericardial effusion. Circulation 1966;34: 611-9. 